\title{
Front Matter: Volume 11186
}

, "Front Matter: Volume 11186," Proc. SPIE 11186, Advanced Optical Imaging Technologies II, 1118601 (20 November 2019); doi: 10.1117/12.2560743

SPIE. Event: SPIE/COS Photonics Asia, 2019, Hangzhou, China 


\title{
PROCEEDINGS OF SPIE
}

\section{Advanced Optical Imaging Technologies II}

\author{
Xiao-Cong Yuan \\ P. Scott Carney \\ Kebin Shi \\ Michael G. Somekh \\ Editors
}

\section{1-23 October 2019 Hangzhou, China}

\author{
Sponsored by \\ SPIE \\ COS-Chinese Optical Society
}

\section{Cooperating Organizations}

Tsinghua University (China) • Peking University (China) • University of Science and Technology of China (China) • Zhejiang University (China) - Tianjin University (China) - Beijing Institute of Technology (China) • Beijing University of Posts and Telecommunications (China) - Nankai University (China) - Changchun University of Science and Technology (China) University of Shanghai for Science and Technology (China) • Capital Normal University (China) • Huazhong University of Science and Technology (China) - Beijing Jiaotong University (China) • China Jiliang University (China) • Shanghai Institute of Optics and Fine Mechanics, CAS (China) - Changchun Institute of Optics, Fine Mechanics and Physics, CAS (China) Institute of Semiconductors, CAS (China) • Institute of Optics and Electronics, CAS (China) • Institute of Physics, CAS (China) Shanghai Institute of Technical Physics, CAS (China) - China Instrument and Control Society (China) • Japan Optical Society (Japan) - Korea Optical Society (Korea, Republic of) - Australia Optical Society (Australia) • Singapore Optical Society (Singapore) • European Optical Society

\section{Supporting Organizations}

China Association for Science and Technology (CAST)

Department of Information of National Nature Science Foundation, China (NSFC)

Published by

SPIE

\section{Volume 11186}


The papers in this volume were part of the technical conference cited on the cover and title page. Papers were selected and subject to review by the editors and conference program committee. Some conference presentations may not be available for publication. Additional papers and presentation recordings may be available online in the SPIE Digital Library at SPIEDigitalLibrary.org.

The papers reflect the work and thoughts of the authors and are published herein as submitted. The publisher is not responsible for the validity of the information or for any outcomes resulting from reliance thereon.

Please use the following format to cite material from these proceedings:

Author(s), "Title of Paper," in Advanced Optical Imaging Technologies II, edited by Xiao-Cong Yuan, P. Scott Carney, Kebin Shi, Michael G. Somekh, Proceedings of SPIE Vol. 11186 (SPIE, Bellingham, WA, 2019) Seven-digit Article CID Number.

ISSN: 0277-786X

ISSN: 1996-756X (electronic)

ISBN: 9781510630895

ISBN: 9781510630901 (electronic)

Published by

SPIE

P.O. Box 10, Bellingham, Washington 98227-0010 USA

Telephone +1 3606763290 (Pacific Time) · Fax + 13606471445

SPIE.org

Copyright @ 2019, Society of Photo-Optical Instrumentation Engineers.

Copying of material in this book for internal or personal use, or for the internal or personal use of specific clients, beyond the fair use provisions granted by the U.S. Copyright Law is authorized by SPIE subject to payment of copying fees. The Transactional Reporting Service base fee for this volume is $\$ 21.00$ per article (or portion thereof), which should be paid directly to the Copyright Clearance Center (CCC), 222 Rosewood Drive, Danvers, MA 01923. Payment may also be made electronically through CCC Online at copyright.com. Other copying for republication, resale, advertising or promotion, or any form of systematic or multiple reproduction of any material in this book is prohibited except with permission in writing from the publisher. The CCC fee code is 0277$786 \times / 19 / \$ 21.00$.

Printed in the United States of America by Curran Associates, Inc., under license from SPIE.

Publication of record for individual papers is online in the SPIE Digital Library.

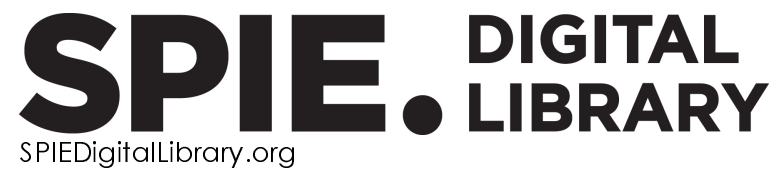

Paper Numbering: Proceedings of SPIE follow an e-First publication model. A unique citation identifier (CID) number is assigned to each article at the time of publication. Utilization of CIDs allows articles to be fully citable as soon as they are published online, and connects the same identifier to all online and print versions of the publication. SPIE uses a seven-digit CID article numbering system structured as follows:

- The first five digits correspond to the SPIE volume number.

- The last two digits indicate publication order within the volume using a Base 36 numbering system employing both numerals and letters. These two-number sets start with $00,01,02,03,04$, 05, 06, 07, 08, 09, OA, OB ... 0Z, followed by 10-1Z, 20-2Z, etc. The CID Number appears on each page of the manuscript. 


\title{
Contents
}

\author{
vii Authors \\ ix Symposium Committees \\ xiii Conference Committee
}

SESSION 1 SUPER-RESOLUTION IMAGING

1118603 Fluorescence optical super-resolution imaging of nitrogen-vacancy centers based on saturated competition microscopy [1 1186-2]

1118605 High-resolution reconstruction method of segmented planar imaging based on compressed sensing [1 $11186-4]$

1118606 Improved microsphere-assisted super-resolution imaging by plasmon coupling [11186-31]

SESSION 2 LABEL-FREE IMAGING I

$1118607 \quad$ Hole-array spatial light modulation for single-pixel imaging (Invited Paper) [1 $1186-5]$

SESSION $3 \quad$ TOMOGRAPHIC IMAGING AND SUPER-RESOLUTION IMAGING

$111860 \mathrm{D} \quad$ Label-free Rheinberg staining of cells using digital holographic microscopy and spatial light interference microscopy [1 $11186-10]$

11186 OE A tunable 3D optical diffraction tomography system with high measurement accuracy [11 1 186-11]

11186 OF Video-rate isotropic quantitative differential phase contrast microscopy based on colormultiplexed annular illumination [1 $1186-12]$

\section{SESSION 4 LABEL-FREE IMAGING II}

$111860 \mathrm{~J} \quad$ Machine-learning enhanced photoacoustic computed tomography in a limited view configuration [1 $11186-18]$

$11186 \mathrm{ON} \quad$ Multicolor stimulated Raman and fluorescence imaging for investigating lipid metabolism [1 $11186-22]$ 
SESSION 5 IMAGING TECHNOLOGIES I

1118600 Three-dimensional macro-scale micro-structure imaging with deep ultraviolet excitation (Invited Paper) [1 $11186-23]$

SESSION $6 \quad$ IMAGING TECHNOLOGIES II

11186 OT Structured illumination microscopy with modified joint Richardson-Lucy deconvolution [1 $11186-28]$

\section{POSTER SESSION}

11186 OY High-speed in vitro intensity diffraction tomography [1 $11186-33]$

1118610 A method of image restoration technology based on parallel phase diversity algorithm [1 $11186-35]$

$1118611 \quad$ Nonlinear focal modulation microscopy based on interleaved reconstruction [1 $1186-36]$

1118612 Propagation of ultrasound-modulated scattered light in biological tissue by using COMSOL multiphysics [ $111186-37]$

1118613 Super-resolution hyperspectral compressed sampling imaging by push-broom coded aperture [1 $11186-38]$

1118614 A method of Fourier ptychography based on variable aperture scanning [11 186-39]

1118615 Influence of spatial power spectrum pattern gray-level distortion on coherent diffraction imaging reconstruction [ 111 186-40]

1118616 Axial resolution enhancement in light sheet microscopy using mode modulation [1 $1186-41]$

1118617 Analysis of encoding aperture processing error for optical field modulation of compressed sampling spectrum imaging [1 $1186-42]$

$1118618 \quad$ Multi-region processing method for single image deraining [1 $1186-43]$

$111861 \mathrm{~A} \quad$ Optical-phase modulator based on stacked piezoelectric ceramics [1 $1186-45]$

11186 1B Frequency shifting confocal microscopy via azimuthally polarized Bessel-Gaussian beam [1 11 186-46]

11186 1D Research progress of coronary artery calcification based on optical coherence tomography [1 11 186-48] 
$111861 \mathrm{~F} \quad$ Measuring the nano-mechanical properties of normal and cancerous prostate cells using atomic force microscopy [1 $1186-50]$

$111861 G \quad$ Methods on adaptive identification with space light modulate [1 $1186-51]$

$111861 \mathrm{H} \quad$ Numerical investigation of photonic nanojets generated from D-shaped dielectric microfibers [1 11 186-52]

$111861 \mathrm{~K} \quad$ Quantitative phase microscopy based on color-multiplexed single-shot Fourier ptychography [1 11 186-56] 
Proc. of SPIE Vol. $111861118601-6$

Downloaded From: https://www.spiedigitallibrary.org/conference-proceedings-of-spie on 26 Apr 2023 Terms of Use: https://www.spiedigitallibrary.org/terms-of-use 


\section{Authors}

Numbers in the index correspond to the last two digits of the seven-digit citation identifier (CID) article numbering system used in Proceedings of SPIE. The first five digits reflect the volume number. Base 36 numbering is employed for the last two digits and indicates the order of articles within the volume. Numbers start with 00,01, 02, 03, 04, 05, 06, 07, 08, 09, OA, OB...0Z, followed by 10-1Z, 20-2Z, etc.

Artur, Camille, 00

Bandyopadhyay, Sankhyabrata, $1 \mathrm{H}$

Cai, Chuangjian, OJ

Cao, Shuo, OE

Cao, Yurong, 06

Chen, Huasong, 18

Chen, Jiabi, OE

Chen, Mengdan, $1 F$

Chen, Qian, OF, OY, IK

Chen, Tao, $1 \mathrm{~A}$

Chen, Yuchen, 11, 16

Deng, Handi, OJ

Ding, Can, 05

Ding, Qin, 18

Ding, Tianxin, 1A

Eriksen, Jason, 00

Fan, Xin, OD

Fan, Yao, OF

Fan, Yuanyuan, 18

Feng, Jiansong, $1 \mathrm{H}$

Fu, Meicheng, 14, 17

Gu, Guoqiang, $1 \mathrm{H}$

Guo, Jiaming, 00

Guo, Yanfang, 13, 14. 15, 17

Hayasaki, Yoshio, 07

Healy, John J., OD

Hennelly, Bryan M., OD

Huang, Lin, 1D

Ito, Yujiro, 07

Jiang, Lianxiang, 10

Jiang, Yupeng, 10

Kong, Meimei, 1A

Kuang, Cuifang, 03, 0T, 11, 16, 1B

Li, Chuankang, 03

Li, Feiyan, 1A

Li, Jiaji, OY

Li, Mengzhu, 13, 15, 17

Li, Mi, OE

Li, Mingsuo, $1 G$

Li, Qin, 1D

Li, Xiujian, 13, 14, 15, 17

Li, Yuhang, 03

Li, Yunzhe, OY

Liang, Zhongcheng, $1 \mathrm{~A}$

Liu, Danni, 1D

Liu, Hua, $1 G$

Liu, Jiying, 13

Liu, Minshi, 10

Liu, Xinyue, 05
Liv, Xu, 03

Liv, Yana, 1D

Liu, Yong, 1B

Liu, Zhongwei, 10

Luo, Jianwen, $0 \mathrm{~J}$

Luo, Jintao, OT

LV, Xing, OE

Ma, Cheng, OJ

Matlock, Alex, OY

Mayerich, David, 00

Meng, Haoran, 05

Oda, Robert, ON

O'Dwyer, Kevin, OD

Ozeki, Yasuyuki, ON

Pan, Jinghan, 14, 17

Pan, Xiangpeng, OF

Peng, Dongqing, 12

Peng, Zeng, $1 \mathrm{H}$

Qi, Junli, 13, 14, 15, 17

Qi, Peiliang, 12

Qiang, Hao, 18

Qin, Na, 1D

Ruan, Weiwei, $1 \mathrm{~F}$

Sato, Ryo, 07

Shao, Liyang, $1 \mathrm{H}$

Shen, Xingliang, $1 \mathrm{H}$

Shou, Jingwen, ON

Song, Haiyang, 12

Sun, Jiasong, OF, $1 \mathrm{~K}$

Tang, Wusheng, 13, 14, 15, 17

Tang, Zhengyuan, OD

Tian, Lei, OY

Wang, Bin, 10

Wang, Guanghui, OE

Wang, Jianguo, 06

Wang, Jingbo, 1D

Wang, Wei, 13, 15

Wang, Weizheng, 13

Wang, Xiaochun, 14, 17

Wang, Xiaofeng, 15

Wang, Xuanhao, OJ

Wang, Yuhua, $1 \mathrm{~F}$

Wu, Chebin, 1A

Xiao, Dongrui, $1 \mathrm{H}$

XU, Liu, OT, 16

$X U$, Min, 05

$\mathrm{XU}$, Zhengxiang, 1A

Yang, Hongqin, $1 \mathrm{~F}$

Yang, Songlin, 06 
Yang, Xinfeng, $1 \mathrm{H}$

Ye, Yong-Hong, 06

Yi, Wenjun, 13, 14, 15, 17

Zeng, Jinshu, $1 \mathrm{~F}$

Zhang, Chengfeng, 11, 16

Zhang, Xiangchao, 05

Zhang, Xiaoman, 12

Zhang, Yasong, 18

Zhao, Rui, 1A

Zhou, Liwei, $1 \mathrm{G}$

Zhu, Jubo, 13, 14, 15, 17

Zhu, Lili, 12

Zhu, Mengjun, 13, 14, 15, 17

Zhu, Ronggang, $1 \mathrm{G}$

Zuo, Chao, OF, OY, IK 


\title{
Symposium Committees
}

\author{
General Chairs
}

Jim M. Oschmann, President, SPIE and Ball Aerospace (United States)

Qihuang Gong, President, Chinese Optical Society and Peking University (China)

General Co-chairs

Guangcan Guo, Past President, Chinese Optical Society and University of Science and Technology of China (China)

Zejin Liu, Vice President, Chinese Optical Society and National University of Defense Technology (China)

Technical Program Chairs

Ruxin Li, Vice President, Chinese Optical Society and Shanghai Institute of Optics and Fine Mechanics (China)

Xingde Li, Johns Hopkins University (United States)

Technical Program Co-chairs

Tianchu Li, National Institute of Metrology (China)

Wei Huang, Northwestern Polytechnical University (China)

Ying Gu, Vice President, Chinese Optical Society and PLA General Hospital (China)

Huilin Jiang, Changchun University of Science and Technology (China)

Wenqing Liu, Vice President, Chinese Optical Society, and Anhui Institute of Optics and Fine Mechanics (China)

Guobin Fan, China Academy of Engineering Physics (China)

Suotang Jia, Vice President, Chinese Optical Society, and Shanxi University (China)

Xiaomin Ren, Vice President, Chinese Optical Society, and Beijing University of Posts and Telecommunications (China)

Secretaries-General

Bo Gu, Deputy Secretary General, Chinese Optical Society (China)

Hong Yang, Deputy Secretary General, Chinese Optical Society and Peking University (China) 
Yan Li, Deputy Secretary General, Chinese Optical Society, and Peking University (China)

Daoxin Dai, Zhejiang University (China)

Local Organizing Committee Chair

Xu Liu, Secretary General, Chinese Optical Society and Zhejiang University (China)

Local Organizing Committee Co-chairs

Jianrong Qiu, Zhejiang University (China)

Daoxin Dai, Zhejiang University (China)

Local Secretaries

Wei Xiong, Chinese Optical Society (China)

Qing Yang, Zhejiang University (China)

Local Organizing Committee

Qing Yang, Zhejiang University (China)

Lan Wu, Zhejiang University (China)

Yaocheng Shi, Zhejiang University (China)

Dong Liu, Zhejiang University (China)

Yungui Ma, Zhejiang University (China)

Ke Si, Zhejiang University (China)

Yang Yang, Zhejiang University (China)

Xinyong Dong, China Jiliang University (China)

Le Wang, China Jiliang University (China)

Fei Tong, Chinese Optical Society (China)

Technical Organizing Committee

Mohammad Hossein Asghari, Loyola Marymount University

(United States) and Tachyonics Inc. (United States)

Pablo Benítez, Universidad Politécnica de Madrid (Spain)

Liangcai Cao, Tsinghua University (China)

P. Scott Carney, University of Rochester (United States)

Benyong Chen, Zhejiang University of Science and Technology

(China)

Hongqiang Chen, GE Global Research (United States)

Daoxin Dai, Zhejiang University (China)

Qionghai Dai, Tsinghua University (China)

Qihuang Gong, Peking University (China)

Ying Gu, Chinese PLA General Hospital (China) 
Guang-Can Guo, University of Science and Technology of China (China)

Byoung Seung Ham, Gwangju Institute of Science and Technology (Korea, Republic of)

Sen Han, University of Shanghai for Science and Technology (China) and Suzhou H\&L Instruments, LLC (China)

Zuyuan He, Shanghai Jiao Tong University (China)

Werner H. Hofmann, Technische Universität Berlin (Germany)

Minghui Hong, National University of Singapore (Singapore)

Bahram Jalali, University of California, Los Angeles (United States)

Satoshi Kawata, Osaka University (Japan)

Baojun Li, Jinan University (China)

Ming Li, Institute of Semiconductors, CAS (China)

Ruxin Li, Shanghai Institute of Optics and Fine Mechanics (China)

Xingde Li, Johns Hopkins University (United States)

Jian Liu, PolarOnyx, Inc. (United States)

Tiegen Liu, Tianjin University (China)

Yongfeng Lu, University of Nebraska-Lincoln (United States)

Qingming Luo, Huazhong University of Science and Technology (China)

Gang-Ding Peng, The University of New South Wales (Australia)

Osamu Matoba, Kobe University (Japan)

Min Qiu, Westlake University (China)

Yuji Sano, ImPACT (Japan)

Yunlong Sheng, Université Laval (Canada)

Kebin Shi, Peking University (China)

Tsutomu Shimura, The University of Tokyo (Japan)

Upendra N. Singh, NASA Langley Research Center (United States)

Michael G. Somekh, Shenzhen University (China)

Yuguo Tang, Suzhou Institute of Biomedical Engineering and

Technology (China)

Masahiko Tani, University of Fukui (Japan)

Limin Tong, Zhejiang University (China)

Kazumi Wada, Massachusetts Institute of Technology (United States)

Yongtian Wang, Beijing Institute of Technology (China)

Rongshi Xiao, Beijing University of Technology (China)

Hongxing $\mathrm{Xu}$, Wuhan University (China)

Jianhua Yao, Zhejiang University of Technology (China)

Toru Yoshizawa, Tokyo University of Agriculture and Technology (Japan) and 3D Associates, Inc. (Japan)

Changyuan Yu, The Hong Kong Polytechnic University (Hong Kong, China)

Xiao-Cong Yuan, Shenzhen University (China)

Cunlin Zhang, Capital Normal University (China)

Song Zhang, Purdue University (United States)

Xi-Cheng Zhang, University of Rochester (United States) 
Xinliang Zhang, Wuhan National Laboratory for Optoelectronics (China)

Xuping Zhang, Nanjing University (China)

Zhenrong Zheng, Zhejiang University (China)

Changhe Zhou, Shanghai Institute of Optics and Fine Mechanics (China)

Zhiping Zhou, Peking University (China)

Dan Zhu, Huazhong University of Science and Technology (China)

Ning Hua Zhu, Institute of Semiconductors, CAS (China) 


\title{
Conference Committee
}

\author{
Conference Chairs \\ Xiao-Cong Yuan, Shenzhen University (China) \\ P. Scott Carney, University of Rochester (United States) \\ Kebin Shi, Peking University (China) \\ Michael G. Somekh, Shenzhen University (China) \\ Conference Program Committee \\ Martin J. Booth, University of Oxford (United Kingdom) \\ David J. Brady, Duke University (United States) \\ Ji-Xin Cheng, Boston University (United States) \\ Bruce Z. Gao, Clemson University (United States) \\ Xiang Hao, Zhejiang University (China) \\ Yoshio Hayasaki, Utsunomiya University Center for Optical Research \\ \& Education (Japan) \\ Minghui Hong, National University of Singapore (Singapore) \\ Zhenli Huang, Huazhong University of Science and Technology \\ (China) \\ Cuifang Kuang, Zhejiang University (China) \\ Malgorzata Kujawińska, Warsaw University of Technology (Poland) \\ Puxiang Lai, The Hong Kong Polytechnic University \\ (Hong Kong, China) \\ Byoungho Lee, Seoul National University (Korea, Republic of) \\ Xu Liu, Zhejiang University (China) \\ Zhiwen Liu, The Pennsylvania State University (United States) \\ Wolfgang Osten, Institut für Technische Optik (Germany) \\ Michelle Y. Sander, Boston University (United States) \\ Paul Urbach, Technische Universiteit Delft (Netherlands) \\ Baoli Yao, Xi'an Institute of Optics and Precision Mechanics, CAS \\ (China) \\ Chao Zuo, Nanjing University of Science and Technology (China)

\section{Session Chairs} \\ $1 \quad$ Super-Resolution Imaging \\ Kebin Shi, Peking University (China) \\ 2 Label-Free Imaging I \\ Qing Yang, Zhejiang University (China)
}


3 Tomographic Imaging and Super-Resolution Imaging

Kebin Shi, Peking University (China)

Ming Lei, Xi'an Jiaotong University (China)

$4 \quad$ Label-Free Imaging II

Xiao-Cong Yuan, Shenzhen University (China)

5 Imaging Technologies I

Kebin Shi, Peking University (China)

6 Imaging Technologies II

Xiao-Cong Yuan, Shenzhen University (China) 